\title{
Review
}

Muyao Zhang ${ }^{1}$, Xing Wei $^{2}$, Zhenfei Wang ${ }^{2 *}$

\section{The mechanism of HBx protein to promote the initiation and progression of hepatocellular carcinoma}

DOI: $10.2478 / \mathrm{ii}-2018-0010$

Received January 29, 2018; accepted February 06, 2018; published online May 15, 2018

Abstract: Hepatocellular carcinoma (HCC) is the sixth most common malignancy worldwide and the third most common cause of death from cancer, after lung and stomach cancer. Hepatitis B virus (HBV) infection is closely related to HCC and is a major cause of HCC. HBV is a lysogenic virus of the hepadnavirus family. Its genome presents a slack, ring-like, double-chain structure, containing four open reading frames. The $\mathrm{X}$ region encodes the product $\mathrm{HBV} \mathrm{X}$ protein $(\mathrm{HBx})$, which is a multifunctional regulatory protein that plays an important role in intracellular signal transduction, viral genome replication and transcription, cell proliferation and apoptosis, cell cycle progression, protein degradation, and genetic stability of hepatocytes. This article summarizes the recent research on the mechanism of promotion of initiation and progression of HCC by HBx protein.

Keywords: HBx protein, hepatocellular carcinoma, HBV

\section{Introduction}

HBV infection-caused chronic liver disease accounts for most of the chronic liver diseases, affecting >240 million people around the world [1]. Each year, about 1 million people die from liver cirrhosis and hepatoma caused by chronic HBV infection [2]. HBV infection is mainly prevalent in Asian countries, such as China, Japan, and South Korea [3]. In China, HBV infection is a serious public health problem. It is estimated that up to 93 million people are infected, and HBV-related liver cirrhosis and hepatoma resulted in 300,000 deaths every year [4]. Therefore, it is extremely important to study the mechanism of promotion of initiation and progression of hepatoma by HBV. HBx protein is one of the products of the HBV genome. Recent studies have found that HBx protein has great effects on the biological behaviors of hepatocytes and hepatoma cells, and that it plays important roles in promoting the initiation and progression of hepatoma, thus holding promise to becoming a new molecular target in hepatocellular carcinoma (HCC) therapy.

\section{HBx protein}

The $\mathrm{X}$ protein $(\mathrm{HBx})$ encoded by the $\mathrm{X}$ region of the open reading frame in the HBV genome is a soluble multifunctional $17 \mathrm{kDa}$ protein composed of 154 amino acids [5]. It is named " $\mathrm{X}$ " as no known protein has been found with homology to it. It has wide trans-activation functions that activate multiple regulatory genes of $\mathrm{HBV}$ and the infected cells [5]. HBx is distributed in the cytoplasm, nucleus, and mitochondria of infected hepatocytes and mainly located in the cytoplasm. HBx in different locations plays different biological functions [6]. It plays an important role in viral genome replication, transcription, DNA repair, protein degradation, and so on. It is widely involved in many cellular signaling pathways and promotes the initiation and progression of HCC [7].

${ }^{1}$ Department of Traditional Chinese Medicine, Inner Mongolia Medical University, Inner Mongolia, China

${ }^{2}$ Affiliated People's Hospital, Inner Mongolia Medical University, Inner Mongolia, China

*Correspondence: Zhenfei Wang, 42 Zhaowuda raod, Huhhot 010020. E-mail: ndwzf2013@126.com 


\section{Mechanisms of the pathological functions of HBx protein}

\subsection{HBx protein promotes the malignant transformation of hepatocytes}

During the initiation and progression of HCC, microRNAs (miRNAs or miRs) miR-16/15a act as tumorsuppressive molecules [8]. Their overexpression can inhibit cell proliferation, clonal formation, and anchorage-independent growth abilities. Their silence can promote cell cycle progression and cell growth. After transfection into hepatocytes, HBx protein upregulates the expression of c-myc, which downregulates the expression of miR-16/15 and then induces a series of malignant behaviors, such as proliferation, migration, and invasion of hepatocytes [8]. It can be seen that changing miRNA expression is an important mechanism by which HBx protein promotes the malignant transformation of hepatocytes.

Alpha-fetoprotein (AFP) is a protein expressed early in HCC. Xia et al. [9] found that in the HBx-induced hepatocyte malignant transformation process, HBx promoted the expression of AFP in hepatocytes. The AFP then stimulated the expression of some protooncogenes in hepatocytes by activating the phosphatidylinositol-3 kinase (PI3K)/protein kinase -A (AKT) signaling pathway. Subsequently, intracellular apoptosis signals were inhibited and growth signals were activated, resulting in the survival of the HBV-infected hepatocytes in the body [9]. In addition, the HBx-induced overexpression of AFP in hepatocytes can stimulate the body to produce inflammatory cytokines, especially interleukin-6 (IL-6). During the process of chronic inflammation stimulation, IL-6 induces the reprogramming of hepatocytes to liver stem cells through an autocrine effect. Considering that AFP also activates the PI3K/AKT signal to stimulate the expression of some protooncogenes, HBx may induce the transformation of liver stem cells to cancer stem cells [9].

\subsection{HBx protein promotes the proliferation of hepatoma cells}

The tumor-suppressive gene p16 is a negative regulator of cell cycle and cell growth. It encodes a cyclin kinase inhibitor, which decreases the phosphorylation level of retinoblastoma $(\mathrm{Rb})$ protein by binding to and inhibiting the cell cycle-dependent protein kinases CDK4 and CDK6. The silencing of p16 gene expression can lead to the unlimited proliferation of cells [10]. Kang et al. found that transfection of HBx into HepG2 cells induced methylation of some $\mathrm{CpG}$ sites in the promoter region of the tumor-suppressive gene $p 16$, then downregulated the expression of $p 16$ gene, and ultimately significantly enhanced the proliferation ability of hepatoma cells [11]. Xu et al. found that HBx and the C-terminal 50-amino acid-deletion mutation of HBx promoted the proliferation of hepatoma cells and that this promoting effect might be mediated by inhibition of the expression of $p 16$ gene. Thus, the amino terminal of HBx protein may be an important functional area for $\mathrm{HBx}$ to regulate the cell cycle and promote the proliferation of hepatoma cells [12].

The Wnt/ $\beta$-catenin pathway is involved in many physiological processes, such as embryonic development. Its abnormal activation leads to initiation and progression of HCC by promoting cell proliferation and increasing the levels of angiogenesis factors, such as matrix metalloproteinase 2 (MMP2) and vascular endothelial growth factor (VEGF)-A [13]. Zheng et al. found that HBx protein can upregulate both mRNA and protein levels of $\mathrm{b}$-catenin in hepatoma cells, activate the $\mathrm{Wnt} / \beta$-catenin pathway, and thus promote the proliferation of hepatoma cells [14].

The HBx protein also acts on the mitochondria in the hepatocyte. It has been reported that HBx protein can change the structure of the calcium ion channel on the membrane of mitochondria to increase the concentration of calcium ion in the cytoplasm, thus initiating the proliferation of HBV [15]. Duan et al. found that HBx protein can increase intracellular oxidative stress and activate the transcription factors signal transducer and activator of transcription (STAT3), nuclear factor kappa B (NFKB), and other factors by causing mitochondrial damage, which results in the acceleration of hepatoma cell proliferation [15].

Long noncoding RNA high expression in HCC (lncRNA-HEIH) is a carcinogenic lncRNA overexpressed in hepatoma. It accelerates the malignant progression of hepatoma by promoting the abnormal proliferation of hepatoma cells [16]. Hu et al. found that HBx can promote the proliferation of hepatoma cells by enhancing the expression of IncRNA-HEIH [16]. 


\subsection{HBx protein inhibits apoptosis of hepatoma cells}

Zhu et al. cotransfected HBx and RNA polymerase II subunit 5 (RPB5)-mediated protein (RMP) into Hep G2 cells and found that the cells coexpressing the two factors showed remarkably decreased apoptosis rate; significantly downregulated expression of Bax, caspase3, and p53; and significantly upregulated expression of the apoptosis inhibitory gene Bcl-2 [17].

There are three main unfolded protein response pressure sensors during endoplasmic reticulum (ER) stress, namely, protein kinase R (PKR)-like ER kinase, inositol-requiring enzyme $1 \alpha$, and transcription factor activating transcription factor 6 (ATF6) [18]. HBx protein directly interacts with glucose-regulated protein $78 \mathrm{kD}$ (GRP78) in ER, which inhibits eukaryotic translation initiation factor 2 (eIF2) alpha phosphorylation and ATF4/CCAAT-enhancer binding protein homologous protein (CHOP)/B-cell lymphoma 2 (Bcl-2) expression, and decreases polyribosomal poly(ADP-ribose) polymerase 1 (PARP-1) and gamma H2AX expression, thereby reducing hepatoma cell apoptosis.

The amino acid transporter gene is a target gene of miR-122. The overexpression of cationic amino acid transporter 1 (CAT-1) gene can inhibit the apoptosis of hepatocytes. HBx can upregulate CAT-1 expression by downregulating miR-122 expression and, ultimately, inhibit the apoptosis of hepatocytes infected with HBV [19].

\subsection{HBx protein promotes the migration and metastasis of hepatoma cells}

Epithelial-mesenchymal transition (EMT) is an important biological process for the proliferation and invasion of malignant tumor cells derived from epithelial cells. Lv and Zhao showed that HBx transfection induced EMT in Hep G2 cells by increasing the activity of cellular sarcoma (c-Src), thereby promoting the migration and invasion of hepatoma cells [20]. Transfecting HBx into SMMC7721 cells significantly upregulated p-NF- $\mathrm{kB}$, p-janus kinase (JAK)2, and p-STAT3 levels and thus activated the JAK/STAT signaling pathway. This effect increased the expression level of programmed death-ligand 1 (PD-L1) on the cell surface, induced EMT in hepatoma cells, and enhanced the migration and invasion abilities of hepatoma cells [21]. A study also found that HBx may play a role in HCC through PD-L1. HBx influences cell viability, migration, and invasion of HCC via PD-L1 HCC cells [22].

Carcinoembryonic antigen-related cell adhesion molecule 1 (CEACAM1), a transmembrane glycoprotein, is a member of the carcinoembryonic antigen (CEA) family. CEACAM1 acts as a tumor-suppressive gene in the initiation and progression of HBV-related HCC. Ma et al. found that HBx promotes the invasion and metastasis of hepatoma cells by inhibiting the expression of CEACAM1 [23].

HBV X-interacting protein (HBXIP) is a highly conserved protein that is widely expressed in adult tissues and is named for its interaction with the HBx protein [24]. The study of Zhang et al. showed that HBXIP is highly expressed in HCC tissues, and its expression is positively related to that of HBx. HBx significantly increases the protein expression of HBXIP by enhancing the activity of its promoter, which promotes the metastasis of hepatoma cells [24].

Zhang et al. found that downregulated expression of F-actin-capping protein subunit beta (CAPZB) in HCC was related to the deletion of the carboxy terminal of HBx protein and correlated with intrahepatic metastasis [25]. Carboxy-terminal-truncated $\mathrm{HBx}$ protein (Ct-HBx) can promote the formation and metastasis of HCC by suppressing CAPZB expression in HCC, which suggests that CAPZB may be involved in the mechanism of Ct-HBx-mediated HCC progression.

\subsection{HBx protein stabilizes HBV DNA in hepatoma cells}

The expression of male-specific lethal 2 (MSL2) protein has been proved to be positively related to HBV infection and is related to the signal pathway associated with viral infection. MSL2 can regulate the level of APOBEC3B ubiquitination by acting as an E3 ubiquitination ligase, which promotes its degradation in hepatoma cells and thus enhances the stability of HBV covalently closed circular DNA (cccDNA) [26]. Gao et al. found that HBx upregulated the transcription of MSL2 gene by promoting the binding of FoxA1 to MSL2 promoter area in hepatocytes. The upregulated expression of MSL2 considerably increased the stability of 
HBV cccDNA, promoted the HBV life cycle, and accelerated the progression of HCC [26]. Interfering with MSL2 expression effectively inhibited the proliferation of hepatoma cells in vivo and in vitro. Therefore, $\mathrm{HBx}$ protein can promote the progression of HCC by enhancing the stability of HBV cccDNA in HCC cells through activation of the positive feedback loop of HBx/MSL2/HBV cccDNA/HBV [26].

\section{Conclusion}

HBx protein plays an important role in the initiation and progression of HBV-related HCC. Elucidating the action mechanism of HBx is very important to explore the pathological mechanism of HCC. The development of new antihepatoma strategies and novel antihepatoma drugs targeting HBx protein will be of huge benefit to the therapy of HCC.

Acknowledgments: This project was supported by the Program for Young Talents of Science and Technology in Universities of Inner Mongolia Autonomous Region NJYT-17-B30.

Conflict of interest: The authors state no conflicts of interest.

Authors' Contributions: Muyao Zhang and Xing Wei conducted the literature analysis and wrote, discussed, and revised the manuscript of this review. Zhenfei Wang critically analyzed and corrected the manuscript. All authors read and approved the final manuscript.

\section{References}

[1] Ott J.J., Stevens G.A., Groeger J., Wiersma S.T., Global epidemiology of hepatitis B virus infection: new estimates of age-specific HBsAg seroprevalence and endemicity, Vaccine, 2012, 30, 212-219.

[2] Revill P., Testoni B., Ocarnini S., Zoulim F., Global strategies are required to cure and eliminate HBV infection, Nat. Rev. Gastroenterol. Hepatol., 2016, 13, 239-248.

[3] Wang M., Xi D., Ning Q., Virus-induced hepatocellular carcinoma with special emphasis on HBV, Hepatol. Int., 2017, 11, 171-180.

[4] Lu F.M., Li T., Liu S., Zhuang H., Epidemiology and prevention of hepatitis B virus infection in China, J. Viral Hepat., 2010, 17(s1), 4-9.

[5] Yu X.H., Yan Y.H., Dong C.H., Li J.F., Li B.G., Yang J.J., et al., The mechanism of HBx in HBV related hepatocellular carcinoma, Gunangdong Med. J., 2016, 37(23), 3614-3618.

[6] Bouchard M.J., Navas-Martin S., Hepatitis B and C virus hepatocarcinogenesis: lessons learned and future challenges, Cancer Lett., 2011, 305(2), 123-143.

[7] Geng M., Xin X., Bi L.Q., Zhou L.T., Liu X.H., Molecular mechanism of hepatitis B virus X protein function in hepatocarcinogenesis, World J. Gastroenterol., 2015, 21(38), 10732.

[8] Wu G., Zheng B., Hunag R., Yang X., HBx promotes hepatoma cell malignant transformation via repressing miR-16 family, Chin. J. Bases Clin. Gen. Surg., 2017, 24(04), 412-419.

[9] Xia H., Zhu M.Y., Li M.S., The effect of HBx induced alpha fetoprotein expression on malignant transformation of hepatocytes and its regulatory mechanism, Shi Jie Hua Ren Xiao Hua Za Zhi, 2015, 23(05), 741-747.

[10] Kotake Y., Naemura M., Murasaki C., Inoue Y., Okamoto H., Transcriptional regulation of the p16 tumor suppressor gene, Anticancer Res., 2015, 35(8), 4397-4401.

[11] Kang Y.H., Li W., Zhan W.L., Shang J., Yang L., Effects of HBV X protein on expression and promoter methylation of p16 tumor suppressor gene, J. Clin. Hepatology, 2016, 32(03), 484-487.

[12] Yao X.B., Yang L., Zhu J.Y., Mai L., Chong Y.T., Effects of wild - type and mutant hepatitis B virus X protein with deletion at C - terminus on cell proliferation in hepatocellular carcinoma J.Gunangdong Med. J., 2015, 36(24), 3742-3745.

[13] Vilchez V., Turcios L., Marti F., Gedaly R., Targeting Wnt/ $\beta$-catenin pathway in hepatocellular carcinoma treatment, World J. Gastroenterol., 2016, 22(2), 823.

[14] Zheng B.Y., Fang X.F., Chen Z.X., Gao W.Y., Li D., Wang X.Z., HBx promotes the proliferation of HepG2 cells through the Wnt/ beta -catenin pathway, J. Fujian Med. Univ., 2016, 50(06), 354-358+364.

[15] Duan C.H., Liu X.C., Dou F.F., Ding J.L., Duan J.F., Zhao X.R., et al., Expression and clinical significance of HBx and Beclin1 in hepatocellular carcinoma, Chin. Hepatol., 2017, 22(01), 20-23. 
[16] Hu J.L., Li L.J., Yi X.M., Lv L., An Y.L., Effect of up-regulating IncRNA-HEIH by HBx on proliferation of hepatocellular carcinoma cells, Chin. J. Hepatic Surg. (Electro. Edn.), 2017, 6(02), 139-143.

[17] Zhu X.X., Wang Q., Li S., He F., Wei W.X., Effect of co-expression of RPB5-mediating protein and hepatitis B virus X protein on the apoptosis of HepG2 cells, Chin. J. Clin. Lab. Sci., 2015, 33(03), 219-222.

[18] Li J., He J., Fu Y.M., Hu X., Sun L.Q., Huang Y., et al., Hepatitis B virus X protein inhibits apoptosis by modulating endoplasmic reticulum stress response, Oncotarget, 2017, 8(56), 96027-96034.

[19] Dai R., Peng F., Xiao X., Gong X., Jiang Y., Zhang M., et al., Hepatitis B virus X protein-induced upregulation of CAT-1 stimulates proliferation and inhibits apoptosis in hepatocellular carcinoma cells, Oncotarget, 2017, 8(37), 60962-60974.

[20] Lv G.B., Zhao Y., HBx protein induces epithelial-mesenchymal transition through c-Src activation in HepG2 cells, J. Shanxi Med. Univ., 2015, 46(12), 1163-1168.

[21] Wang H., Chen L., Tumor microenviroment and hepatocellular carcinoma metastasis, J. Gastroenterol. Hepatol, 2013, 28(S1), 43-48.

[22] Tian J.X., Liu J.L., Liang J., HBx enhances cell viability, migration and invasion of hepatocellular carcinoma via PD-L1, Cancer Cell Res., 2017, 14, 351-355.

[23] Ma L., Zhang W., Xu G.Z., Yao Y., Xu L., Liu K.G., Expressions of HBx and CEACAM1 in HBV-related hepatocellular carcinoma and their significances, J. Jilin Univ. (Med. Edn.), 2012, 4, 060.

[24] Zhang W.Y., Zhang X.D., Ye L.H., Hepatitis B Virus X protein promotes hepatoma cell migration via CREB-induced up-regulation of hepatitis B X-interacting protein, Zhongguo Sheng Wu Hua Xue Yu Fen Zi Sheng Wu Xue Bao, 2015, 31(05), 473-480.

[25] Zhang Q.Q., Li W.H., Li H., Zhang Z.X., Wei J.Y., The expression and clinical significance of carboxyl end truncated hepatitis B virus X protein and cap protein B in liver cancer, Acad. J. Guangzhou Med. Univ., 2017, 45(02), 1-5.

[26] Gao Y., Feng J., Yang G., Zhang S., Liu Y., Bu Y., et al., HBx-elevated MSL2 modulates HBV cccDNA through inducing degradation of APOBEC3B to enhance hepatocarcinogenesis, Hepatology, 2017, 66, 1413-1429. 